\title{
AN ACCURACY ASSESSMENT OF GEOREFERENCED POINT CLOUDS PRODUCED VIA MULTI-VIEW STEREO TECHNIQUES APPLIED TO IMAGERY ACQUIRED VIA UNMANNED AERIAL VEHICLE
}

\author{
Steve Harwin and Arko Lucieer \\ School of Geography and Environmental Studies \\ University of Tasmania \\ Private Bag 76, Hobart, Australia 7001 \\ Stephen.Harwin@utas.edu.au
}

KEY WORDS: Point Cloud, Accuracy, Reference Data, Surface, Georeferencing, Bundle, Reconstruction, Photogrammetry

\begin{abstract}
:
Low-cost Unmanned Aerial Vehicles (UAVs) are becoming viable environmental remote sensing tools. Sensor and battery technology is expanding the data capture opportunities. The UAV, as a close range remote sensing platform, can capture high resolution photography on-demand. This imagery can be used to produce dense point clouds using multi-view stereopsis techniques (MVS) combining computer vision and photogrammetry. This study examines point clouds produced using MVS techniques applied to UAV and terrestrial photography. A multi-rotor micro UAV acquired aerial imagery from a altitude of approximately 30-40 m. The point clouds produced are extremely dense $(<1-3 \mathrm{~cm}$ point spacing) and provide a detailed record of the surface in the study area, a $70 \mathrm{~m}$ section of sheltered coastline in southeast Tasmania. Areas with little surface texture were not well captured, similarly, areas with complex geometry such as grass tussocks and woody scrub were not well mapped. The process fails to penetrate vegetation, but extracts very detailed terrain in unvegetated areas. Initially the point clouds are in an arbitrary coordinate system and need to be georeferenced. A Helmert transformation is applied based on matching ground control points (GCPs) identified in the point clouds to GCPs surveying with differential GPS. These point clouds can be used, alongside laser scanning and more traditional techniques, to provide very detailed and precise representations of a range of landscapes at key moments. There are many potential applications for the UAV-MVS technique, including coastal erosion and accretion monitoring, mine surveying and other environmental monitoring applications. For the generated point clouds to be used in spatial applications they need to be converted to surface models that reduce dataset size without loosing too much detail. Triangulated meshes are one option, another is Poisson Surface Reconstruction. This latter option makes use of point normal data and produces a surface representation at greater detail than previously obtainable. This study will visualise and compare the two surface representations by comparing clouds created from terrestrial MVS (T-MVS) and UAV-MVS.
\end{abstract}

\section{INTRODUCTION}

Terrain and Earth surface representations were traditionally derived from imagery using analogue photogrammetric techniques that produced contours and topological maps from stereo pairs. Digital photogrammetry has sought ways to automate the process and improve efficiency. Modern mesh or grid based representations provide relatively efficient storage of terrain data at a wide range of resolutions. The quality of these representations is dependent on the techniques used for data capture and processing. The representation improves with resolution and the data capture technique must be able to accurately determine height points at sufficient density to portray the shape of the surface. The difficulty faced is that the storage and visualisation become increasingly difficult as resolution increases. The surface must therefore be represented by an approximation that resembles reality as closely as possible.

In recent decades photogrammetric techniques have sought to improve surface representation through automated feature extraction and matching. Computer vision uses Structure from Motion (SfM) to achieve similar outputs. SfM incorporates multiview stereopsis (MVS) techniques that match features in multiple views of a scene and derive 3D model coordinates and camera position and orientation. The Scale Invariant Feature Transform (SIFT) operator (Lowe, 2004) provides a robust description of features in a scene and allows features distinguished in other views to be compared and matched. A bundle adjustment can then be used to derive a set of 3D coordinates of matched features. The point density is proportional to the number of matched features and untextured surfaces, occlusions, illumination changes and acquisition geometry can result in fewer matches (Remondino and El-Hakim, 2006). The Bundler software ${ }^{1}$ is an open source tool for performing least squares bundle adjustment (Snavely et al., 2006). To reduce computing overheads imagery is often down sampled. Typically the next stage is to densify the point cloud using MVS techniques, such as the patch-based multi-view stereo software PMVS2 ${ }^{2}$. Each point in the resulting cloud has an associated normal. The point clouds produced from UAV imagery (referred to as UAV-MVS) acquired at 30-50 m flying height above ground level (AGL) have a density of $\sim 1-3$ points per $\mathrm{cm}^{2}$. There can be in excess of 7 million points in a cloud (file size of $\sim 500 \mathrm{Mb}$ ). The point cloud generated can be georeferenced by matching control points in the cloud to surveyed ground control points (GCPs). The resulting accuracy is dependent on the accuracy of the GCP survey or reference datasets and in this case it is approximately 25-40 mm (Harwin and Lucieer, 2012). The accuracy can be improved with coregistration to a more accurate base dataset.

To allow these large datasets to be used it is usually necessary to convert them into a more storage efficient data structure so that the data can be used in conventional GIS and 3D visualisation software that rely on a surface for texturing rather than a point cloud. Grid based (or Raster) and triangular mesh based data models, such as Digital Surface Models (DSMs) and Triangular Irregular Networks (TINs), are commonly used. After processing and classification a Digital Elevation Model (DEM) or a Digital Terrain Model (DTM) representation of the earth's surface, without any vegetation or man-made structures, can be de-

\footnotetext{
${ }^{1}$ http://phototour.cs.washington.edu/bundler/

${ }^{2} \mathrm{http}$ //grail.cs.washington.edu/software/pmvs/
} 
rived. The process of deriving these surface structures from a set of sample points is traditionally done using computational geometry based methods such Delauney triangulation or the Voronoi diagram (Bolitho et al., 2009). The data is assumed to be free from noise and dense enough to allow a realistic surface to be derived (Zhou et al. (2010) in Lim and Haron (2012)). When the point cloud is sparse or noisy the resulting surface is often jagged rather than smooth. The surface reconstruction process interpolates heights between sample points (Bolitho et al., 2009). Each point is considered a moment of height change and between points terrain height change is assumed to be linear or is solved by interpolating a least squares fit. An alternative to computational geometry is function fitting, these approaches define a function for determining a surface at a given location by global and/or local fitting (Bolitho et al., 2009). Kazhdan et al. (2006) developed a Poisson Surface Reconstruction technique that combines both global and local function fitting expressed as a solution to a Poisson equation (Bolitho et al., 2009). The Poisson approach uses the orientation of the point normal to create a surface that changes gradient according to the change in point orientation (Figure 1). The algorithm obtains a reconstructed surface with greater detail than previously achievable (Jazayeri et al., 2010).

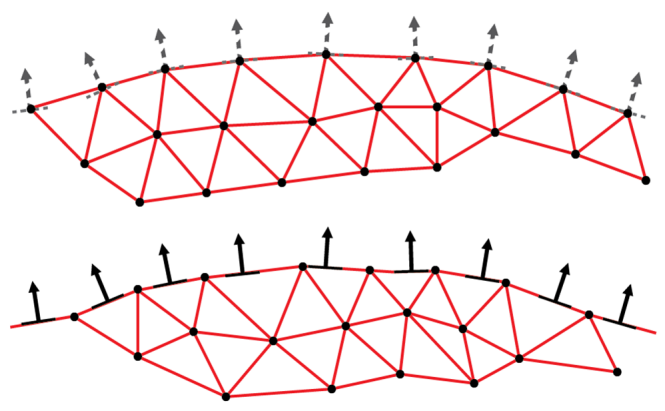

Figure 1: A TIN versus a Poisson DSM.

This paper evaluates the UAV-MVS generated point cloud and surface representations of a natural land form by qualitatively comparing these to a reference dataset generated using close range terrestrial photography based MVS techniques (T-MVS).

\section{METHODS}

\subsection{Study Area}

A dynamic $100 \mathrm{~m}$ section of sheltered estuarine coastline in south eastern Tasmania will be monitored for fine scale change (Figure 2). The vegetation on the site is grasses and scrub bush along an erosion scarp with salt marsh at the southern end of the study site. For this study a section of the erosion scarp was chosen as the focus area for comparing the close range terrestrial MVS point cloud to the UAV-MVS point cloud (Figure 3).

\subsection{Hardware}

The camera chosen to capture photography at sufficient resolution for UAV-MVS point cloud generation is the Canon 550D digital SLR camera. This camera has a light weight camera body and provides control over ISO, aperture and shutter speed settings. The settings are carefully chosen to reduce motion blur when acquiring images at $1 \mathrm{~Hz}$ (one photo per second). The resulting image dataset contains around 300 photographs per UAV flight with 70-95\% overlap. The OktoKopter micro UAV platform (Mikrokopter, 2012) is the basis for the TerraLuma UAV used for this study. The aircraft is an electric multi-rotor system

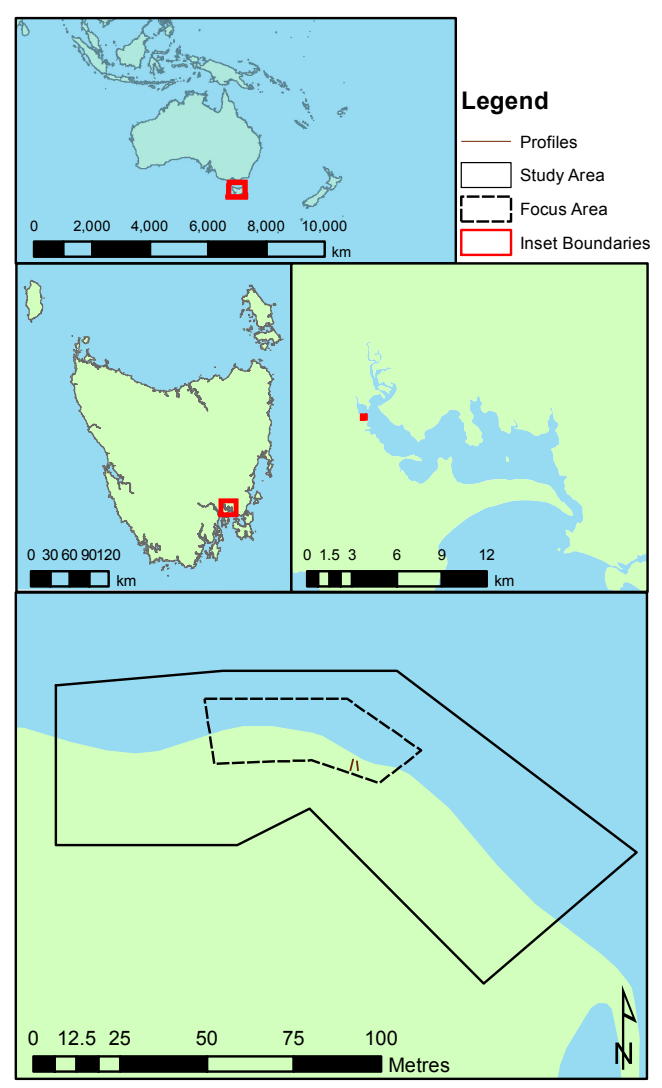

Figure 2: Coastal monitoring site.
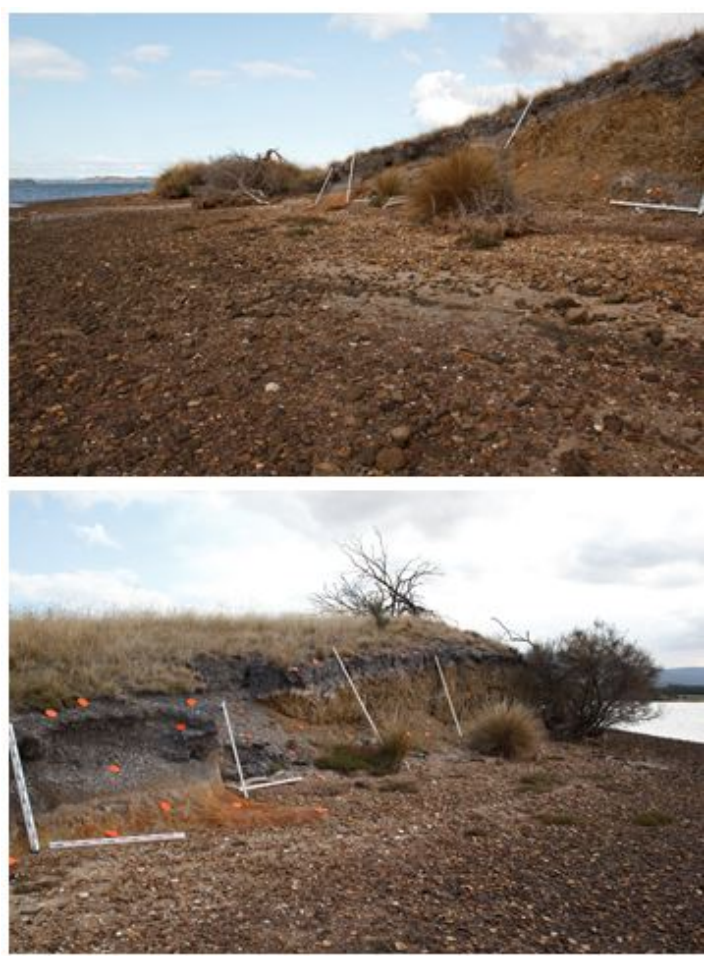

Figure 3: Images of the focus site (the first is taken looking east, the second is taken looking west).

(eight rotors) capable of carrying a $\sim 2.5 \mathrm{~kg}$ payload for approximately six minutes. The system has an on-board GPS (5-10 m positional accuracy) and other sensors that allow it to do waypoint navigation. The camera is attached to a stabilised camera 
mount. This camera is also used for the hand held terrestrial photography. A Leica 1200 real-time kinematic dual-frequency differential GPS (RTK DGPS) was used to capture ground control.

\subsection{Data Collection}

To generate the UAV-MVS point cloud 89 photographs were taken from nadir and 64 oblique photographs were taken from a $\sim 45^{\circ}$ angle. The above ground level (AGL) flying height was approximately $30-40 \mathrm{~m}$. Prior to acquiring the UAV imagery 42 small $10 \mathrm{~cm}$ orange disks were distributed throughout the focus area. These GCP disks were surveyed using RTK DGPS to an accuracy of $\sim 1.5-2.5 \mathrm{~cm}$. These disks were placed so that they could be seen from above and from the waters edge. The UAV imagery captured these GCPs in $~ 380$ overlapping aerial photographs and then 179 terrestrial photographs were taken of the focus area by hand. The UAV image dataset and the terrestrial dataset were carefully screened and any blurred photographs or photographs beyond the study area were rejected.

\subsection{Multi-View Stereopsis}

The MVS process relies on matching features in multiple photographs of a subject, in this case a section of coastline. The Bundler software is used to perform a least squares bundle adjustment on the matched features. These features are discovered and described using invariant descriptor vectors or SIFT feature vectors. Once defined the SIFT features (or in our version SIFTFast features ${ }^{3}$ ) can be matched and the MVS process produces a sparse $3 \mathrm{D}$ point cloud along with the position and orientation of the camera for each image. Radial distortion parameters are also derived. The imagery used in this first step is down sampled (5184x3456 pixels $\Rightarrow 2000 \times 1333$ pixels). The point cloud produced is in an arbitrary coordinate space. The next stage is to densify the point cloud using PMVS2, usually with down sampled images. The improvement made by our UAV-MVS process is that we transform the output from the Bundler bundle adjustment so that PMVS2 can run on the full resolution imagery. The resulting set of 3D coordinates also includes point normals, however it is still in an arbitrary coordinate reference frame.

\subsection{Georeferencing}

The ground control points must identified in the imagery and matched to their GPS positions in the local UTM coordinate system (GDA94 Zone 55). This "semi-automatic GCP georeferencing" is done by analysing the colour attributes of a random selection of orange GCP disks found in the imagery. The point cloud is then filtered based on the derived colour thresholds, i.e. Red, Green, Blue (RGB) range for GCP orange. The filter finds points in the cloud that are close enough in RGB colour space Euclidean distance to the GCP orange. The extracted orange point cloud contains clusters of points for each GCP and the bounding box of those point clusters is used to calculate a cluster centroid for each GCP cluster in the arbitrary coordinate space. To match these cluster centres to the equivalent surveyed GPS positions, the navigation grade on-board GPS positions for the time synchronised camera locations are matched to the Bundler derived camera positions and a Helmert Transformation is derived that, when applied, locates the point cloud in real work scale to an accuracy of $\sim 5$ $10 \mathrm{~m}$. The cloud is now in real world scale, therefore the GCP cluster centroid can be matched to the GPS positions by manually finding the closest GCP position to each cluster (when GCPs are more dispersed this process is usually automated). The resulting list of GCP disk cluster centres matched to GCP GPS points is then used to derive new Helmert Transformation parameters for

\footnotetext{
${ }^{3} \mathrm{http}: / /$ sourceforge.net/projects/libsift/
}

transforming from arbitrary coordinate space to the UTM coordinate space.

The terrestrial photography does not have an equivalent set of camera position as the photographs were taken by hand. A "manual GCP georeferencing" technique must therefore be undertaken. This involves to extracting and labelling GCP disk cluster centres from the point cloud and then comparing the distribution to the GPS survey. GPS points can then be matched to their associated cluster centre and a Helmert Transformation can be derived and applied to point clouds and derived surfaces. Once the data was georeferenced it could be clipped into profiles and smaller point clouds using LASTools ${ }^{4}$.

\subsection{Surface Generation}

Triangulated meshes join the points in the dataset to their nearest neighbours, for this study the focus is on the points (or vertices) before and after Poisson Surface Reconstruction (since the vertex locations will remain the same when a dense triangulated mesh is created). Poisson surface reconstruction was done using Version 3 of the PoissonRecon software ${ }^{5}$ provided by Michael Kazhdan and Matthew Bolitho. Default settings were used for all parameters except octree depth and solver divide, for which the values of 12 and 8, respectively, were chosen based on experimentation. MeshLab ${ }^{6}$ and Eonfusion ${ }^{7}$ were used to visualise point clouds and surfaces and clean the data. Edge face removal using length thresholds were used as well as isolated piece removal (automated and manual). The mesh vertices were then extracted by clipping out the profiles (using LASTools) for comparison with the original MVS derived vertex profiles.

\subsection{Point Cloud and Surface Comparison}

Future studies will investigate the best methods for quantitatively comparing point clouds and derived surfaces. For this study the method chosen was a qualitative comparison of point cloud profiles and strips along lines of interest within the focus area (see Figure 4).

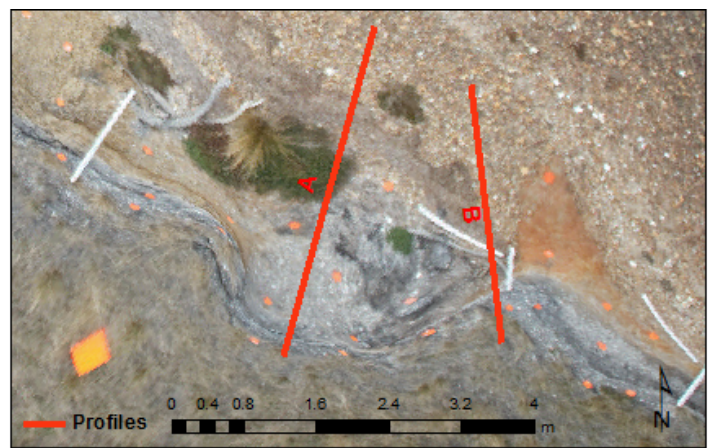

Figure 4: The two profiles within the focus area (see Figure 2).

Profile strips 1, 2 and $6 \mathrm{~cm}$ wide were extracted from the georeferenced MVS point clouds and from the Poisson vertex points clouds. The points and derived surfaces were then overlaid and visually compared to evaluate how well the Poisson vertices represent the surface and how well the UAV-MVS point cloud coincides with the T-MVS point clouds and derived Poisson vertices and surface meshes.

\footnotetext{
${ }^{4} \mathrm{http}: / /$ www.cs.unc.edu/ isenburg/lastools/

${ }^{5}$ http://www.cs.jhu.edu/ misha/Code/PoissonRecon/

${ }^{6}$ http://meshlab.sourceforge.net/

${ }^{7}$ http://www.eonfusion.com/
} 


\section{RESULTS AND DISCUSSION}

The MVS workflow was applied to the terrestrial and the UAV image datasets. For the UAV-MVS dataset, 151 of 153 images chosen were processed resulting in a point cloud $\sim 175 \mathrm{~m}$ by $\sim 60 \mathrm{~m}$ containing $\sim 7.3$ million points $\left(\sim 1-3\right.$ points per $\left.\mathrm{cm}^{2}\right)$. For the T-MVS dataset, 174 of 179 images chosen were processed resulting in a point cloud $\sim 175 \mathrm{~m}$ by $\sim 60 \mathrm{~m}$ containing $\sim 6.3$ million points $\left(\sim 3-5\right.$ points per $\left.\mathrm{cm}^{2}\right)$. Screen shots of these two clouds and close up views of two $1 \mathrm{~m}$ staves are shown in Figure 3.

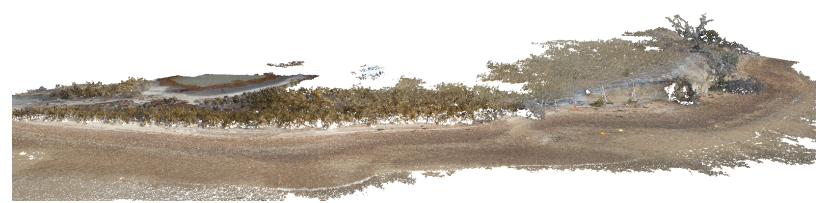

(a) The UAV-MVS point cloud.

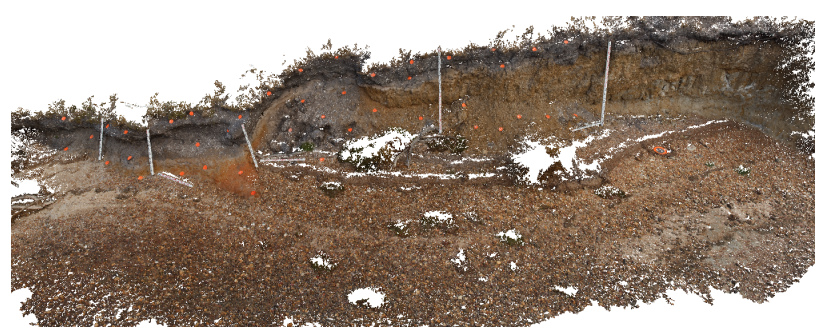

(b) The T-MVS point cloud.

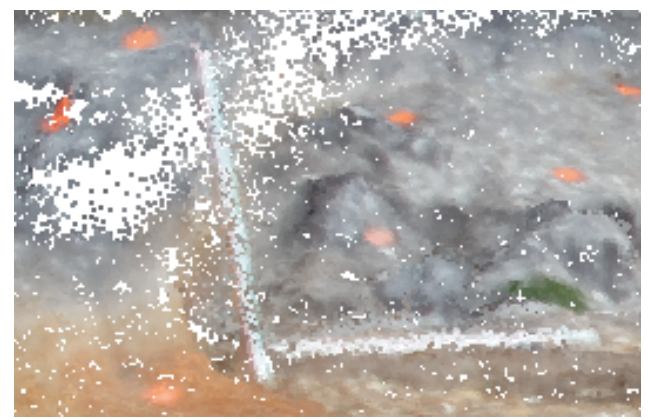

(c) The close up view of the UAV-MVS point cloud (point size $=2$ ).

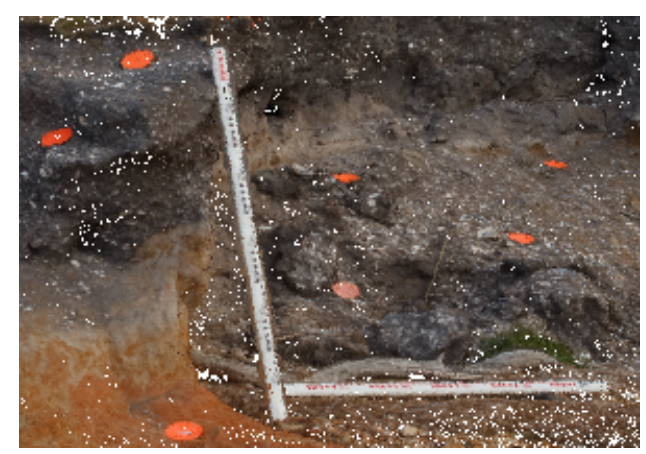

(d) The close up view of the T-MVS point cloud (point size $=1)$.

Figure 5: The derived point clouds.

Both point clouds have sparse sections in the woody scrub bush, dead bushes and longer grasses. The UAV-MVS dataset has more points representing vegetation in the central portion of the focus area, this is not surprising due to the occlusion caused by taking the T-MVS photography from the water side of these bushes. Both point clouds have a high density of points on the erosion scarp and for soil and rock in general, even where the scarp is overhanging. The texture of the ground in these areas is ideal for feature identification as there is a lot of rocky gravel and shell grit in the soil and the beach is very pebbly.

To analyse the effect of surface composition on point density profiles were visualised and compared. For illustrative purposes a number of screen shots are provided that show regions or views of interest. The Eonfusion scene is a far better viewing environment than the flat screen shots provided as the view perspective can easily be adjusted to focus on interesting features from various angles.

As can be seen in Figure 6(a) and Figure 7(a) showing profile A the blue UAV-MVS points are amongst or slightly below the T-MVS points. As the profile crosses the vegetation the sparse TMVS points on the occluded side of the bush can be seen amongst the relatively dense UAV-MVS points.

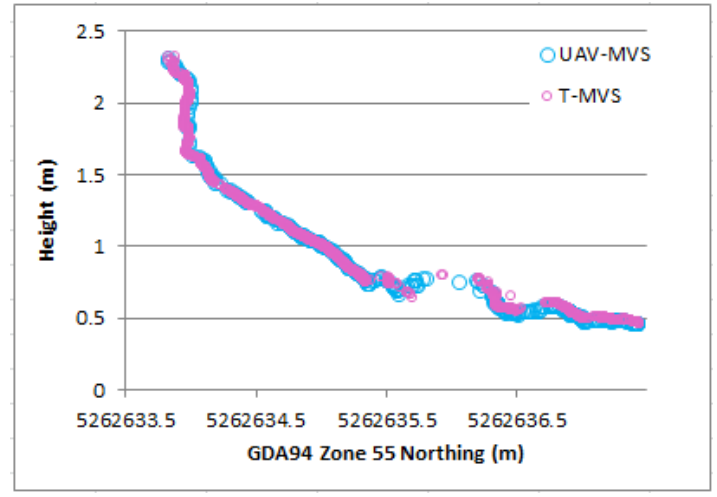

(a) Profile A.

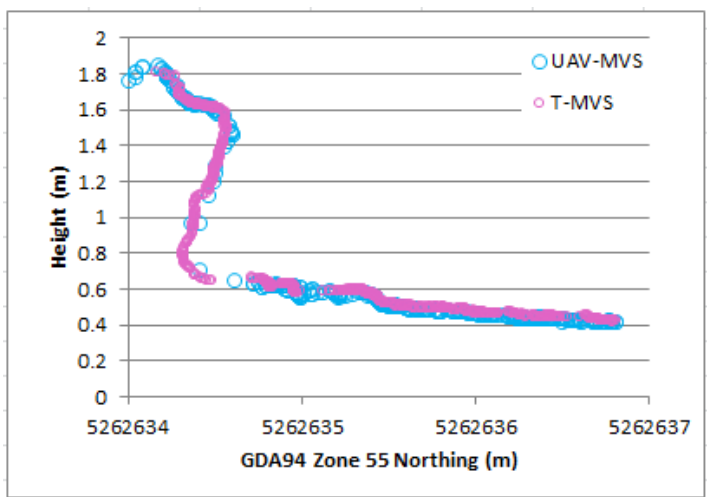

(b) Profile B.

Figure 6: A $1 \mathrm{~cm}$ wide profiles of the UAV-MVS and T-MVS point clouds.

On the pebbly beach the UAV-MVS cloud is consistently below the T-MVS cloud $(<1 \mathrm{~cm})$ (see Figure 6(b) and Figure 7(b)). This may simply be due to differences in the Helmert transformation. Coregistration would be required to assess this further in a future study.

The Poisson surfaces derived from these two clouds produced new point clouds of surface vertices. The UAV-MVS Poisson surface point cloud (referred to as UAV-MVS Poisson) has 2.3 million vertices and the T-MVS Poisson surface point clouds (referred to as T-MVS Poisson) has 1.8 million vertices. After cleaning, the number of vertices were reduced by $\sim 1100$ and $\sim 6000$ points respectively. To visualise and qualitatively assess the effectiveness of the Poisson reconstruction and compare it to the raw point cloud vertices (which would be used to create a dense triangulated mesh surface), the extracted profiles were overlaid and visualised in Eonfusion. 


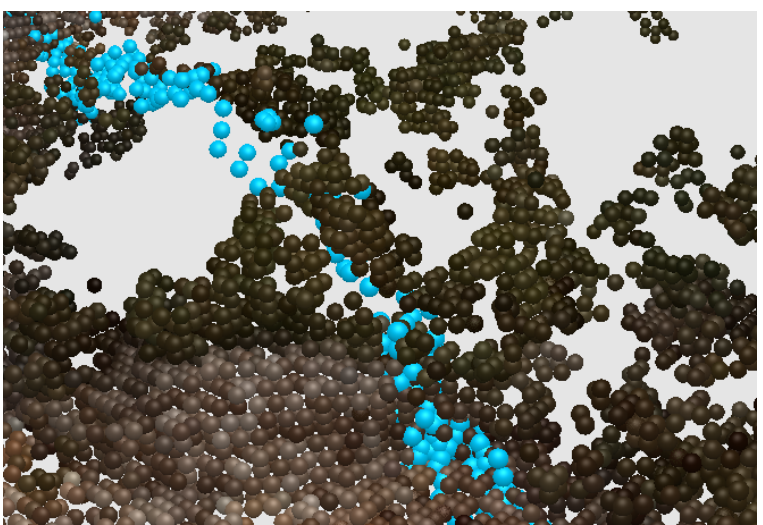

(a) The blue UAV-MVS points amongst the T-MVS points from above.

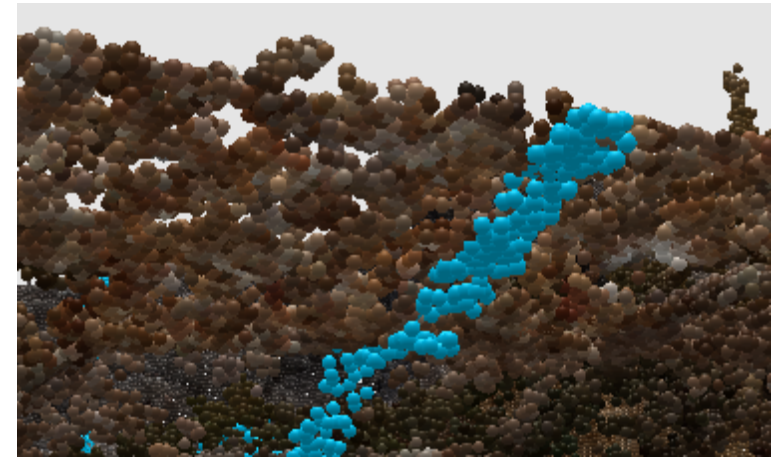

(b) The blue UAV-MVS points beneath the T-MVS points from below.

Figure 7: A $6 \mathrm{~cm}$ wide strip (profile A) of the UAV-MVS point cloud viewed with the T-MVS point cloud.

The wider $6 \mathrm{~cm}$ profile strips have been created as surfaces to assess the difference between UAV-MVS Poisson and T-MVS Poisson. Figure 3 shows the TIN surface compared to the Poisson surface for T-MVS and UAV-MVS datasets respectively.

In these views the natural coloured point clouds have been offset in the $\mathrm{Z}$ dimension by $-10 \mathrm{~cm}$ to allow visualisation of the shape of the surface compared to the cloud that it was derived from. The surface covers a vegetated section and in the UAV-MVS dataset the denser section previously mentioned can be seen when comparing this view (Figure 8(a)) to the same view of the shrub (Figure 8(b)). The T-MVS cloud is sparse here and as a result the Poisson surface seems to have exaggerated the shrub height over the sparse section, probably due to the orientation of the normals varying greatly for those few points, which happens in vegetation. The triangulated mesh is much more jagged than the Poisson surface in both views and the UAV-MVS Poisson is particularly smooth (Figure 8(a)). The shrub in reality does have a reasonably smooth shape, in in this instance the UAV-MVS Poisson appears most accurate. To examine this further a section of Profile B that passes through the pebbly beach is visualised. In Figure 9(b) the Poisson surface is again smoother and the drop in terrain at this point point is well represented (see Figure 6(b)). In Figure 9(a) the same seems evident. In Figure 9(c) the Poisson surfaces for UAV-MVS and T-MVS are shown on the T-MVS point cloud (Z$10 \mathrm{~cm}$ ). In this view the UAV-MVS surface is again $\sim 1 \mathrm{~cm}$ below the T-MVS surface, but the shape of the terrain is basically the same, where as when two raw MVS based TINs are compared in the same view the outliers in the UAV-MVS data seem to cause the surface to vary suddenly causing spikes or peaks in terrain that are not evident in the equivalent T-MVS TIN.

These visualisations provide insight into the quality of terrain and

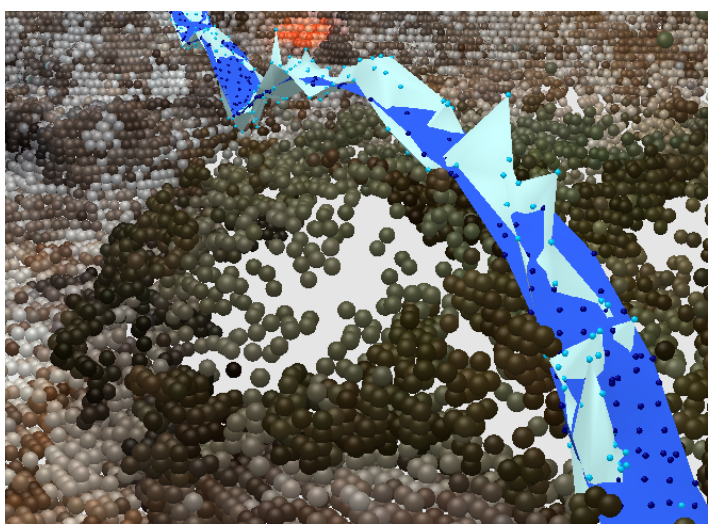

(a) The UAV-MVS point cloud below Poisson (blue) and TIN (light blue) strips.

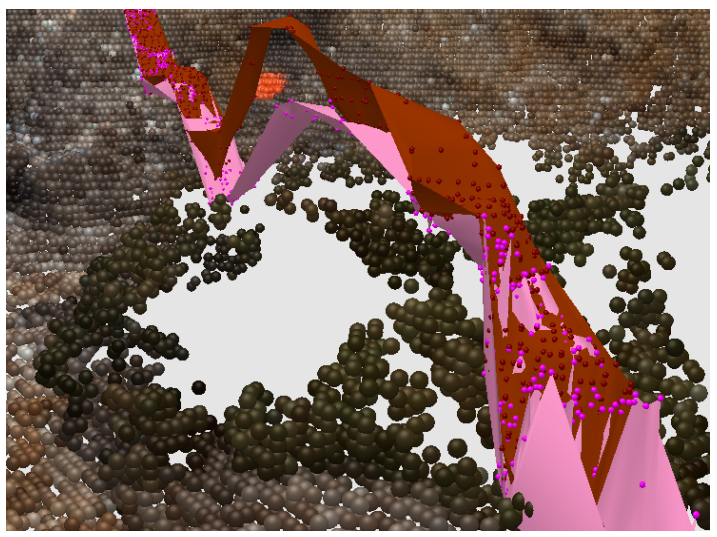

(b) The T-MVS point cloud below Poisson (brown) and TIN (pink) strips.

Figure 8: $6 \mathrm{~cm}$ wide strips of Poisson and TIN surfaces viewed over a vegetated section of the points clouds from which they were derived $(\mathrm{Z}-10 \mathrm{~cm})$, each natural coloured dot has a $14 \mathrm{~mm}$ diameter.

surface extraction possible using MVS techniques. The use of Poisson surface reconstruction has potential advantages over traditional triangulated mesh creation. Poisson surfaces seem generally smoother and smooth surface representations are often better when undertaking decimation, hydrological analysis, DEM derivative extraction and vegetation and ground filtering. The apparent outliers in the point cloud may not impact on the outputs from these analyses and, provided the point cloud density is carefully monitored and taken into account when mapping surface quality, the result may be more realistic for most surface types. Some vegetated areas have complex geometry (such as complex overlapping branches or tussock grasses) and areas with little or no texture are going to be poorly represented and this may impact on the Poisson reconstruction. The creation of a TIN is still a viable option, particularly when the point cloud can be maintained without decimation. When products with a smaller memory footprint are required there seems to be a strong case for using Poisson surface reconstruction to create a fairly smooth yet detailed representation of the terrain from which lower resolution surfaces can be extracted. The TIN surfaces appear more jagged and these spikes in the terrain can cause erroneous height values in a derived output.

\section{CONCLUSIONS AND FUTURE WORK}

This study presented a qualitative assessment of the accuracy of point clouds derived using multi-view stereo techniques (MVS). 


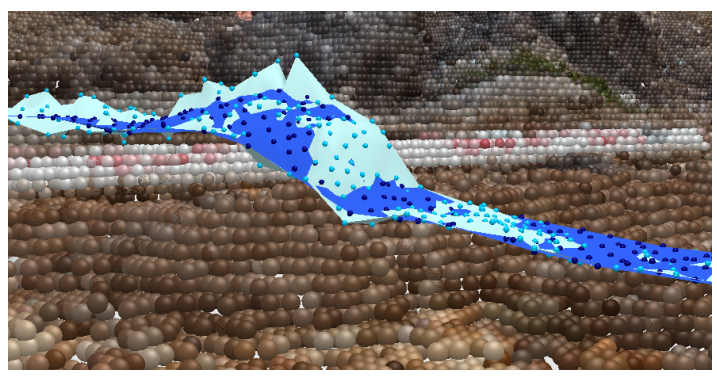

(a) The UAV-MVS point cloud below Poisson (blue) and TIN (light blue) strips.

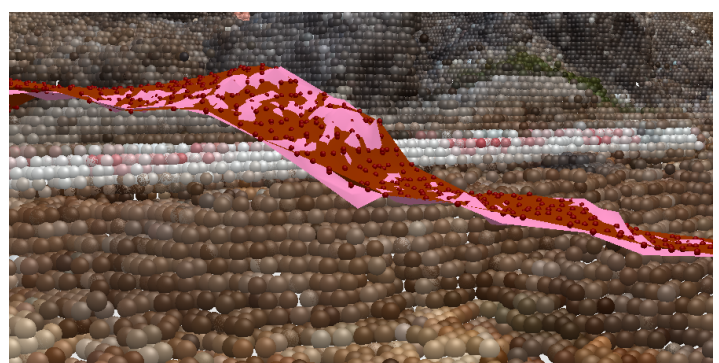

(b) The T-MVS point cloud below Poisson (brown) and TIN (pink) strips.

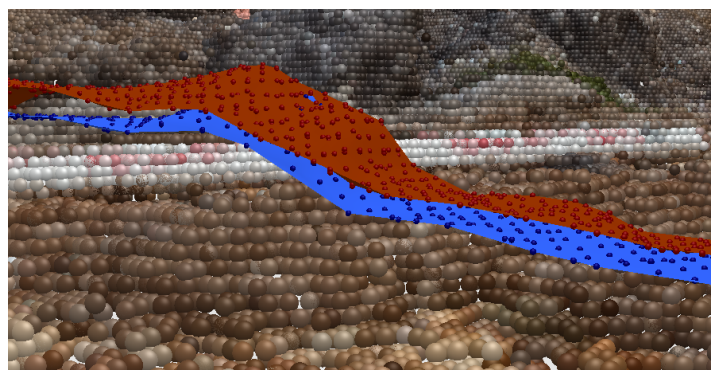

(c) The T-MVS point cloud below UAV-MVS Poisson (blue) and T-MVS Poisson (brown) strips.

Figure 9: $6 \mathrm{~cm}$ wide strips of Poisson and TIN surfaces viewed over a pebbly beach section of the points clouds from which they were derived $(\mathrm{Z}-10 \mathrm{~cm})$, each natural coloured dot has a $14 \mathrm{~mm}$ diameter.

Two datasets were derived using the technique, one using terrestrial photography and the other using photography acquired via an unmanned aerial vehicle (UAV). The two point clouds provided dense point coverage of the areas captured in the imagery, the terrestrial MVS dataset had $\sim 3-5$ points per $\mathrm{cm}^{2}$ and the UAV-MVS dataset had $\sim 1-3$ points per $\mathrm{cm}^{2}$. Once georeferenced the two clouds coincided quite well, however in future studies comparison will be between coregistered datasets. Triangulated meshing and Poisson surface reconstruction was used to create surface models and these models were compared and evaluated to assess how well the terrain and surface features were portrayed. The point clouds produced using MVS have point normals associated with each point and this allows detailed surface features to be derived using Poisson surface reconstruction. The derivatives that can be extracted from such a detailed surface representation will benefit from the Poisson algorithm as it combines global and local function fitting and seems to smooth the data and the process is not strongly influenced by outliers in the point cloud. Future studies will undertake quantitative assessment of the differences and evaluate the potential of these techniques for change detection, in this study area the fine scale coastal erosion that is occurring may be indicative of climate change and, if this technique proves useful, UAVs may be a viable tool for focussed monitoring studies. The issues faced in vegetated areas and areas with complex geometry that result in sparse patches in the point cloud need to be investigated, it may be that the key areas of change are still well represented. The MVS technique has a great deal of potential both in natural and man-made landscapes and there are many potential applications for the use of UAVs for remote sensing data capture, alongside laser scanning and more traditional techniques, to provide very detailed and precise representations of a range of landscapes at key moments. Application areas include landform monitoring, mine surveying and other environmental monitoring. Qualitatively, the outputs from the UAV-MVS process compare very well to the terrestrial MVS results. The UAV can map a greater area faster and from more viewing angles, it is therefore an ideal platform for capturing very high detail 3D snapshots of these environments.

\section{ACKNOWLEDGEMENTS}

The authors would like to thank Darren Turner for his logistical, technical and programming support and UAV training. Thank you to Myriax for the scholarship license for the Eonfusion nD spatial analysis package. In addition, for making their algorithms and software available, we would like to give thanks and appreciation to Noah Snavely and his team for Bundler, David Lowe for SIFT, the libsift team for SIFTFast, Yasutuka Furukawa and Jean Ponce for their multi-view stereopsis algorithms (PMVS2 and CMVS) and Martin Isenburg for LASTools.

\section{References}

Bolitho, M., Kazhdan, M. and Burns, R., 2009. Parallel poisson surface reconstruction. Advances in Visual Computing LNCS 5875, pp. 678-689.

Harwin, S. and Lucieer, A., 2012. Assessing the accuracy of georeferenced point clouds produced via multi-view stereopsis from Unmanned Aerial Vehicle (UAV) imagery. Currently in review, publication pending.

Jazayeri, I., Fraser, C. and Cronk, S., 2010. Automated 3D Object Reconstruction Via Multi-Image Close-Range Photogrammetry. International Archives of Photogrammetry, Remote Sensing and Spatial Information Sciences XXXVIII(2000), pp. 38.

Kazhdan, M., Bolitho, M. and Hoppe, H., 2006. Poisson surface reconstruction. In: Proceedings of the Eurographics Symposium on Geometry Processing.

Lim, S. and Haron, H., 2012. Surface reconstruction techniques: a review. Artificial Intelligence Review.

Lowe, D. G., 2004. Distinctive Image Features from ScaleInvariant Keypoints. International Journal of Computer Vision 60(2), pp. 91-110.

Mikrokopter, 2012. "MikroKopter WiKI" http://www.mikrokopter.com.

Remondino, F. and El-Hakim, S., 2006. Image-based 3D Modelling: A Review. The Photogrammetric Record 21(115), pp. 269-291.

Snavely, N., Seitz, S. M. and Szeliski, R., 2006. Photo Tourism: Exploring image collections in 3D. ACM Transactions on Graphics 25(3), pp. 835.

Zhou, K., Gong, M., Huang, X. and Guo, B., 2010. Data-Parallel Octrees for Surface Reconstruction. IEEE transactions on visualization and computer graphics 17(5), pp. 669-681. 